\title{
SYMMETRIZATION OF JORDAN DIALGEBRAS
}

\author{
MURRAY R. BREMNER
}

\begin{abstract}
A basic problem for any class of nonassociative algebras is to determine the polynomial identities satisfied by the symmetrization and the skewsymmetrization of the original product. We consider the symmetrization of the product in the class of special Jordan dialgebras. We use computational linear algebra to show that every polynomial identity of degree $n \leq 5$ satisfied by the symmetrized Jordan diproduct in every diassociative algebra is a consequence of commutativity. We determine a complete set of generators for the polynomial identities in degree 6 which are not consequences of commutativity. We use a constructive version of the representation theory of the symmetric group to show that there exist further new identities in degree 7.
\end{abstract}

\section{INTRODUCTION}

Let $\mathcal{V}$ be a class of nonassociative algebra: 1 over a field with a single bilinear operation denoted $a \cdot b$ which is neither commutative nor anticommutative. For background on the theory of varieties of nonassociative algebras defined by polyomial identities, we refer to Osborn's long paper 21, the "Russian book" 24, and the monographs on Jordan algebras by Jacobson [10] and McCrimmon [20].

For an algebra $A \in \mathcal{V}$ we define two other operations on the underlying vector space, one commutative and one anticommutative: the symmetrized product (anticommutator, Jordan product) $\{a, b\}=a \cdot b+b \cdot a$, and the skew-symmetrized product (commutator, Lie bracket) $[a, b]=a \cdot b-b \cdot a$. The resulting algebras are denoted $A^{+}$and $A^{-}$, and called the plus and minus algebras of $A$. This process is closely related to the polarization of operations studied by Markl \& Remm [18.

For a given class $\mathcal{V}$, a basic problem in nonassociative algebra is to determine the polynomial identities satisfied by all algebras of the form $A^{+}$, respectively $A^{-}$. In the most familiar case, the variety of associative algebras, it is known that:

- Every algebra $A^{+}$satisfies the Jordan identity, and every polynomial identity of degree $n \leq 7$ satisfied by every $A^{+}$follows from commutativity and the Jordan

2010 Mathematics Subject Classification. Primary 17A30. Secondary 16W10, 17A50, 17C05, 17C50, 18D50, 68W30.

Key words and phrases. Special Jordan dialgebras, diassociative algebras, symmetrization, polynomial identities, computational linear algebra, representation theory of the symmetric group.

The author's research was supported by the Discovery Grant Algebraic Operads from NSERC, the Natural Sciences and Engineering Research Council of Canada. He thanks Vladimir Dotsenko for introducing him to the problem of polynomial identities satisfied by bilinear operations in diassociative algebras. This work was completed in November 2017 during the author's visit to Suleyman Demirel University in Kaskelen, Kazakhstan. He thanks Askar Dzhumadildaev and Nurlan Ismailov, and their colleagues and students, for providing a stimulating research environment.

${ }^{1}$ We interpret "nonassociative" loosely to mean "not necessarily associative". 
identity. However, there are "special" identities of degree $n \geq 8$ which are satisfied by every $A^{+}$but which are not consequences of commutativity and the Jordan identity. The simplest of these were discovered by Glennie [9].

- Every algebra $A^{-}$satisfies the Jacobi identity, and the Poincaré-Birkhoff-Witt theorem implies that every polynomial identity of every degree satisfied by every $A^{+}$is a consequence of anticommutativity and the Jacobi identity.

Definition 1.1. Loday [14,15] introduced the notion of diassociative algebra (or associative dialgebra), which is a vector space with two bilinear operations $\vdash$ and $\dashv$, the right and left products, satisfying the following polynomial identities:

$$
\begin{array}{ll}
(x \vdash y) \vdash z \equiv x \vdash(y \vdash z), & (x \dashv y) \dashv z \equiv x \dashv(y \dashv z), \\
(x \vdash y) \dashv z \equiv x \vdash(y \dashv z), & \\
(x \dashv y) \vdash z \equiv(x \vdash y) \vdash z, & x \dashv(y \dashv z) \equiv x \dashv(y \vdash z) .
\end{array}
$$

These are right, left, and inner associativity, and the left and right bar identities.

In the setting of diassociative algebras, the analogues of the Jordan product and the Lie bracket are the Jordan diproduct (antidicommutator) $\{a, b\}=\widehat{a} b+b \widehat{a}$ and the Leibniz bracket (dicommutator) $[a, b]=\widehat{a} b-b \widehat{a}$. It is known that:

- The Jordan diproduct in every diassociative algebra satisfies polynomial identities in degrees 3 and 4 which define the variety of Jordan dialgebras: see Kolesnikov [1], Velásquez \& Felipe [22], Bremner [4]. Every identity of degree $n \leq 7$ follows from these identities. However, there are "special" identities in degree 8 which are not consequences of these identities: the simplest were discovered by Bremner \& Peresi [8]; see also Voronin [23], Kolesnikov \& Voronin [12].

- The Leibniz bracket satisfies the derivation identity in degree 3 which defines the variety of Leibniz algebras; see Loday [13. A generalization of the PoincaréBirkhoff-Witt theorem implies that every polynomial identity of every degree satisfied by the Leibniz bracket in every diassociative algebra is a consequence of this identity; see Loday \& Pirashvili [16], Aymon \& Grivel [1], Bokut et al. [2].

Since the Jordan diproduct and the Leibniz bracket are neither commutative nor anticommutative, it is a basic problem to determine the polynomial identities satisfied by the algebras $A^{+}$and $A^{-}$where $A$ is a Jordan dialgebra or a Leibniz algebra. This paper studies the algebras $A^{+}$where $A$ is a Jordan dialgebra.

Definition 1.2. A (left) Jordan dialgebra is a vector space with a bilinear operation denoted $(x, y) \mapsto x y$ satisfying the following polynomial identities:

$$
x \cdot(y \cdot z) \equiv x \cdot(z \cdot y), \quad(y \cdot x) \cdot x^{2} \equiv\left(y \cdot x^{2}\right) \cdot x, \quad\left(y, x^{2}, z\right) \equiv 2(y, x, z) \cdot z,
$$

where $x^{2}=x \cdot x$ and $(x, y, z)=(x \cdot y) \cdot z-x \cdot(y \cdot z)$ is the associator.

We are concerned with the polynomial identities satisfied by the symmetrization of the Jordan diproduct in every diassociative algebra:

$$
x y=x \cdot y+y \cdot x=\widehat{x} y+\widehat{y} x+y \widehat{x}+x \widehat{y} .
$$

We work over a field of characteristic 0, unless otherwise indicated. This assumption implies that every polynomial identity is equivalent to a finite set of multilinear identities [24, Chapter 1], so we may apply the representation theory of the symmetric group 6]. Some large computations require arithmetic modulo a prime $p$ to reduce memory usage. Since the structure constants for the group algebra $\mathbb{Q} S_{n}$ have denominators which are divisors of $n$ !, if we use a prime $p>n$ where $n$ is 
the degree of the identities, then the group algebra $\mathbb{F}_{p} S_{n}$ is semisimple and we can apply rational reconstruction to recover the results in characteristic 0 . We do not distinguish between the polynomial identity $f \equiv g$ and the polynomial $f-g$.

\section{Algebraic Operads}

The results of this paper may be conveniently formulated in the language of algebraic operads 15,17,19. The operads we consider are symmetric operads in the symmetric monoidal category of vector spaces over a field of characteristic 0 ; the product is the tensor product, and the coproduct is the direct sum.

Definition 2.1. We write Free for the free (symmetric) operad generated by a commutative (nonassociative) binary operation $\omega$. We write Dias for the (symmetrization of the nonsymmetric) diassociative operad generated by the right and left operations $\rho$ and $\lambda$. A morphism $X$ : Free $\longrightarrow$ Dias is uniquely determined by its action on $\omega$; we therefore define the expansion map by

$$
X(\omega)=\lambda+\lambda^{(12)}+\rho+\rho^{(12)} .
$$

The right side of this equation is the operadic form of the symmetrized Jordan diproduct where the superscript permutations act on the arguments.

Lemma 2.2. The following dimension formulas are well-known:

$$
\operatorname{dim} \operatorname{Free}(n)=(2 n-3) ! ! \text { where } n ! !=\prod_{i=0}^{\lfloor n / 2\rfloor}(n-2 i), \quad \operatorname{dim} \operatorname{Dias}(n)=n(n !) .
$$

Remark 2.3. The number of inequivalent association types (placements of parentheses) for a commutative nonassociative operation is given by the sequence of Wedderburn-Etherington numbers [3], which begins $1,1,1,2,3,6,11, \ldots$.

Algorithm 2.4. Loday [14,15 proved that the diassociative identities imply a simple normal form for diassociative monomials $m=\overline{x_{1} \cdots x_{n}}$ where the overline indicates an arbitrary placement of parentheses and an arbitrary assignment of right and left operation symbols. We express $m$ as a plane rooted complete binary tree $t$ with $n$ leaves labelled $x_{1}, \ldots, x_{n}$ from left to right and $n-1$ internal nodes (including the root) labelled by operation symbols. Starting at the root, we follow the path determined by the operations: $\vdash$ or $\dashv$ indicate respectively that we choose the right or left subtree. This path terminates at a unique leaf $x_{i}$, called the center (or middle) of the diassociative monomial $m$. It follows that

$$
m=x_{1} \vdash \cdots \vdash x_{i-1} \vdash x_{i} \dashv x_{i+1} \dashv \cdots \dashv x_{n},
$$

and that this expression is independent of the placement of parentheses. This allows us to omit the operation symbols and denote the center by a hat:

$$
m=x_{1} \cdots x_{i-1} \widehat{x}_{i} x_{i+1} \cdots x_{n} .
$$

Multiplication of monomials then takes the following simple form where the direction of the operation symbol determines the center of the product:

$$
\begin{aligned}
& x_{1} \cdots \widehat{x}_{i} \cdots x_{p} \vdash y_{1} \cdots \widehat{y}_{j} \cdots y_{q}=x_{1} \cdots x_{i} \cdots x_{p} y_{1} \cdots \widehat{y}_{j} \cdots y_{q}, \\
& x_{1} \cdots \widehat{x}_{i} \cdots x_{p} \dashv y_{1} \cdots \widehat{y}_{j} \cdots y_{q}=x_{1} \cdots \widehat{x}_{i} \cdots x_{p} y_{1} \cdots y_{j} \cdots y_{q}
\end{aligned}
$$


Example 2.5. In degree 3, we have the following ordered monomial bases:

Free(3): $(a b) c,(a c) b,(b c) a ; \quad \operatorname{Dias}(3): \widehat{a^{\sigma}} b^{\sigma} c^{\sigma}, a^{\sigma} \widehat{b^{\sigma}} c^{\sigma}, a^{\sigma} b^{\sigma} \widehat{c^{\sigma}}\left(\sigma \in S_{3}\right)$.

(The elements of $S_{3}$ are in lex order.) The following formula for $X((a b) c)$ is easily verified, and permutation of the arguments gives $X((a c) b)$ and $X((b c) a)$ :

$$
\widehat{a} b c+\widehat{b} a c+2 \widehat{c} a b+2 \widehat{c} b a+a \widehat{b} c+b \widehat{a} c+c \widehat{a} b+c \widehat{b} a+2 a b \widehat{c}+2 b a \widehat{c}+c a \widehat{b}+c b \widehat{a} .
$$

We obtain (the transpose of) the matrix representing $X$ in degree 3 (dot for zero):

$$
\left[\begin{array}{llllllllllllllllll}
1 & . & 1 & . & 2 & 2 & 1 & . & 1 & . & 1 & 1 & 2 & . & 2 & . & 1 & 1 \\
. & 1 & 2 & 2 & 1 & . & . & 1 & 1 & 1 & 1 & . & . & 2 & 1 & 1 & 2 & . \\
2 & 2 & . & 1 & . & 1 & 1 & 1 & . & 1 & . & 1 & 1 & 1 & . & 2 & . & 2
\end{array}\right]
$$

This matrix has rank 3 , so every identity in degree 3 follows from commutativity.

\section{DegreEs 4 AND 5}

Lemma 3.1. Every multilinear polynomial identity of degree $n \leq 4$ satisfied by the symmetrization of the Jordan diproduct is a consequence of commutativity.

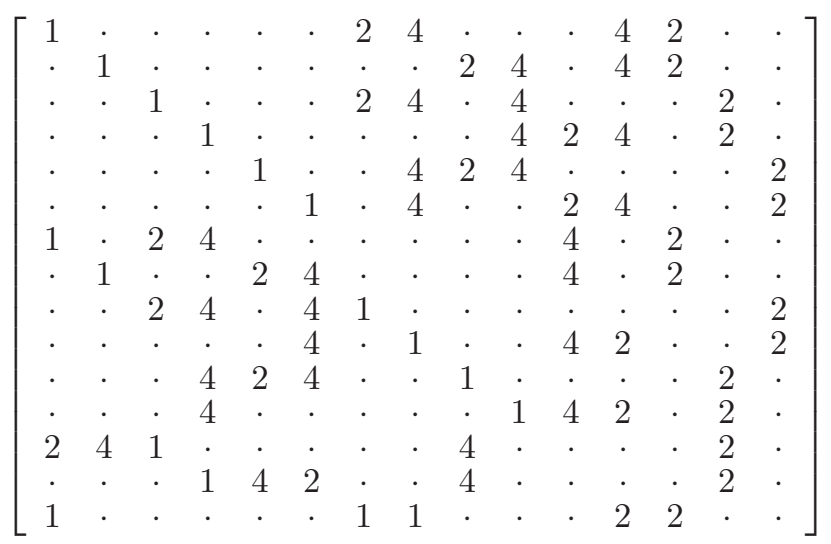

Figure 1. Full rank submatrix for proof of Lemma 3.1

Proof. Similar to Example2.5, but the matrix is larger. In degree 4, the two association types $((--)-)-$ and $(--)(--)$ for a commutative nonassociative operation have respectively 12 and 3 multilinear monomials, ordered by type and then by permutation of the arguments. We expand the monomials with the identity permutation into the diassociative operad using the symmetrized Jordan diproduct:

$$
\begin{aligned}
& X(((a b) c) d)=\widehat{a} b c d+\widehat{b} a c d+2 \widehat{c} a b d+2 \widehat{c} b a d+4 \widehat{d a} a b c+4 \widehat{d b} a c+4 \widehat{d} c a b+4 \widehat{d} c b a \\
& +a \widehat{b} c d+b \widehat{a} c d+c \widehat{a} b d+c \widehat{b} a d+d \widehat{a} b c+d \widehat{d} a c+2 d \widehat{c} a b+2 d \widehat{c} b a \\
& +2 a b \widehat{c} d+2 b a \widehat{c} d+c a \widehat{b} d+c b \widehat{a} d+d a \widehat{b} c+d b \widehat{a} c+d c \widehat{a} b+d c \widehat{b} a \\
& +4 a b c \widehat{d}+4 b a c \widehat{d}+4 c a b \widehat{d}+4 c b a \widehat{d}+2 d a b \widehat{c}+2 d b a \widehat{c}+d c a \widehat{b}+d c b \widehat{a}, \\
& X((a b)(c d))=2 \widehat{a} b c d+2 \widehat{a} b d c+2 \widehat{b} a c d+2 \widehat{b} a d c+2 \widehat{c} d a b+2 \widehat{c} d b a+2 \widehat{d} c a b+2 \widehat{d c} b a \\
& +2 a \widehat{b} c d+2 a \widehat{b} d c+2 b \widehat{a} c d+2 b \widehat{a} d c+2 c \widehat{d a} b+2 c \widehat{d b} a+2 d \widehat{c} a b+2 d \widehat{c} b a \\
& +2 a b \widehat{c} d+2 a b \widehat{d c}+2 b a \widehat{c} d+2 b a \widehat{d c}+2 c d \widehat{a} b+2 c c \widehat{d b} a+2 d c \widehat{a} b+2 d c \widehat{b} a \\
& +2 a b c \widehat{d}+2 a b d \widehat{c}+2 b a c \widehat{d}+2 b a d \widehat{c}+2 c d a \widehat{b}+2 c d b \widehat{a}+2 d c a \widehat{b}+2 d c b \widehat{a} \text {. }
\end{aligned}
$$


Permutation of the arguments gives the expansions of the other monomials. In degree 4 , we order the $4 \cdot 4 !=96$ multilinear diassociative monomials by position of the center and then by permutation of the arguments. We construct the $96 \times 15$ matrix $E$ whose $(i, j)$ entry is the coefficient of the $i$-th diassociative monomial in the expansion of the $j$-th commutative nonassociative monomial. The submatrix consisting of rows $1-14$ and 25 corresponds to the diassociative monomials $\widehat{a} b c d, \ldots$, $\widehat{c} a d b, a \widehat{b} c d$ (Figure 1). This submatrix has full rank which completes the proof.

$$
\begin{aligned}
& X((((a b) c) d) e)= \\
& \widehat{a} b c d e+\widehat{b} a c d e+2 \widehat{c} a b d e+2 \widehat{c} b a d e+4 \widehat{d} a b c e+4 \widehat{d b} a c e+4 \widehat{d} c a b e+4 \widehat{d} c b a e \\
& +8 \widehat{e} a b c d+8 \widehat{e} b a c d+8 \widehat{e} c a b d+8 \widehat{e} c b a d+8 \widehat{e} d a b c+8 \widehat{e} d b a c+8 \widehat{e} d c a b+8 \widehat{e} d c b a \\
& +a \widehat{b} c d e+b \widehat{a} c d e+c \widehat{a} b d e+c \widehat{b} a d e+d \widehat{a} b c e+d \widehat{b} a c e+2 d \widehat{c} a b e+2 d \widehat{c} b a e \\
& +e \widehat{a} b c d+e \widehat{b} a c d+2 e \widehat{c} a b d+2 e \widehat{c} b a d+4 e \widehat{d a} b c+4 e \widehat{d b} a c+4 e \widehat{d} c a b+4 e \widehat{d} c b a \\
& +2 a b \widehat{c} d e+2 b a \widehat{c} d e+c a \widehat{b} d e+c b \widehat{a} d e+d a \widehat{b} c e+d b \widehat{a} c e+d c \widehat{a} b e+d c \widehat{b} a e \\
& +e a \widehat{b} c d+e b \widehat{a} c d+e c \widehat{a} b d+e c \widehat{b} a d+e d \widehat{a} b c+e \widehat{d b} a c+2 e d \widehat{c} a b+2 e d \widehat{c} b a \\
& +4 a b c \widehat{d e}+4 b a c \widehat{d e}+4 c a b \widehat{d e}+4 c b a \widehat{d e}+2 d a b \widehat{c} e+2 d b a \widehat{c} e+d c a \widehat{b} e+d c b \widehat{a} e \\
& +2 e a b \widehat{c} d+2 e b a \widehat{c} d+e c a \widehat{b} d+e c b \widehat{a} d+e d a \widehat{b} c+e d b \widehat{a} c+e d c \widehat{a} b+e d c \widehat{b} a \\
& +8 a b c d \widehat{e}+8 b a c d \widehat{e}+8 c a b d \widehat{e}+8 c b a d \widehat{e}+8 d a b c \widehat{e}+8 d b a c \widehat{e}+8 d c a b \widehat{e}+8 d c b a \widehat{e} \\
& +4 e a b c \widehat{d}+4 e b a c \widehat{d}+4 e c a b \widehat{d}+4 e c b a \widehat{d}+2 e d a b \widehat{c}+2 e d b a \widehat{c}+e d c a \widehat{b}+e d c b \widehat{a}, \\
& X(((a b)(c d)) e)= \\
& 2 \widehat{a} b c d e+2 \widehat{a} b d c e+2 \widehat{b} a c d e+2 \widehat{b} a d c e+2 \widehat{c} d a b e+2 \widehat{c} d b a e+2 \widehat{d} c a b e+2 \widehat{d} c b a e \\
& +8 \widehat{e} a b c d+8 \widehat{e} a b d c+8 \widehat{e} b a c d+8 \widehat{e} b a d c+8 \widehat{e} c d a b+8 \widehat{e} c d b a+8 \widehat{e} d c a b+8 \widehat{e} d c b a \\
& +2 a \widehat{b} c d e+2 a \widehat{b} d c e+2 b \widehat{a} c d e+2 b \widehat{a} d c e+2 c \widehat{d a} b e+2 c \widehat{d b} a e+2 d \widehat{c} a b e+2 d \widehat{c} b a e \\
& +2 e \widehat{a} b c d+2 e \widehat{a} b d c+2 e \widehat{b} a c d+2 e \widehat{b} a d c+2 e \widehat{c} d a b+2 e \widehat{c} d b a+2 e \widehat{d} c a b+2 e \widehat{d c} b a \\
& +2 a b \widehat{c} d e+2 a b \widehat{d} c e+2 b a \widehat{c} d e+2 b a \widehat{d} c e+2 c d \widehat{a} b e+2 c d \widehat{b} a e+2 d c \widehat{a} b e+2 d c \widehat{b} a e \\
& +2 e a \widehat{b} c d+2 e a \widehat{b} d c+2 e b \widehat{a} c d+2 e b \widehat{a} d c+2 e c \widehat{d} a b+2 e c \widehat{d b} a+2 e d \widehat{c} a b+2 e d \widehat{c} b a \\
& +2 a b c \widehat{d e}+2 a b d \widehat{c} e+2 b a c \widehat{d e}+2 b a d \widehat{c} e+2 c d a \widehat{b} e+2 c d b \widehat{a} e+2 d c a \widehat{b} e+2 d c b \widehat{a} e \\
& +2 e a b \widehat{c} d+2 e a b \widehat{d} c+2 e b a \widehat{c} d+2 e b a \widehat{d} c+2 e c d \widehat{a} b+2 e c d \widehat{b} a+2 e d c \widehat{a} b+2 e d c \widehat{b} a \\
& +8 a b c d \widehat{e}+8 a b d c \widehat{e}+8 b a c d \widehat{e}+8 b a d c \widehat{e}+8 c d a b \widehat{e}+8 c d b a \widehat{e}+8 d c a b \widehat{e}+8 d c b a \widehat{e} \\
& +2 e a b c \widehat{d}+2 e a b d \widehat{c}+2 e b a c \widehat{d}+2 e b a d \widehat{c}+2 e c d a \widehat{b}+2 e c d b \widehat{a}+2 e d c a \widehat{b}+2 e d c b \widehat{a}, \\
& X(((a b) c)(d e))= \\
& 2 \widehat{a} b c d e+2 \widehat{a} b c e d+2 \widehat{b} a c d e+2 \widehat{b} a c e d+4 \widehat{c} a b d e+4 \widehat{c} a b e d+4 \widehat{c} b a d e+4 \widehat{c} b a e d \\
& +4 \widehat{d e} a b c+4 \widehat{d e} b a c+4 \widehat{d e} c a b+4 \widehat{d e} c b a+4 \widehat{e} d a b c+4 \widehat{e} d b a c+4 \widehat{e} d c a b+4 \widehat{e} d c b a \\
& +2 a \widehat{b} c d e+2 a \widehat{b} c e d+2 b \widehat{a} c d e+2 b \widehat{a} c e d+2 c \widehat{a} b d e+2 c \widehat{a} b e d+2 c \widehat{b} a d e+2 \widehat{c} \hat{b} a e d \\
& +4 d \widehat{e} a b c+4 d \widehat{e} b a c+4 d \widehat{e} c a b+4 d \widehat{e} c b a+4 e \widehat{d a} b c+4 e \widehat{d b} a c+4 e \widehat{d} c a b+4 e \widehat{d c} b a \\
& +4 a b \widehat{c} d e+4 a b \widehat{c} e d+4 b a \widehat{c} d e+4 b a \widehat{c} e d+2 c a \widehat{b} d e+2 c a \widehat{b} e d+2 c b \widehat{a} d e+2 c b \widehat{a} e d \\
& +2 d e \widehat{a} b c+2 d e \widehat{b} a c+4 d e \widehat{c} a b+4 d e \widehat{c} b a+2 e d \widehat{a} b c+2 e d \widehat{b} a c+4 e d \widehat{c} a b+4 e d \widehat{c} b a \\
& +4 a b c \widehat{d e}+4 a b c \hat{e} d+4 b a c \widehat{d e}+4 b a c \widehat{e} d+4 c a b \widehat{d e}+4 c a b \widehat{e} d+4 c b a \widehat{d e}+4 c b a \widehat{e} d \\
& +2 d e a \widehat{b} c+2 d e b \widehat{a} c+2 d e c \widehat{a} b+2 d e c \widehat{b} a+2 e d a \widehat{b} c+2 e d b \widehat{a} c+2 e d c \widehat{a} b+2 e d c \widehat{b} a \\
& +4 a b c d \widehat{e}+4 a b c e \widehat{d}+4 b a c d \widehat{e}+4 b a c e \widehat{d}+4 c a b d \widehat{e}+4 c a b e \widehat{d}+4 c b a d \widehat{e}+4 c b a e \widehat{d} \\
& +4 d e a b \widehat{c}+4 d e b a \widehat{c}+2 d e c a \widehat{b}+2 d e c b \widehat{a}+4 e d a b \widehat{c}+4 e d b a \widehat{c}+2 e d c a \widehat{b}+2 e d c b \widehat{a} \text {. }
\end{aligned}
$$

FIGURE 2. Expansions with identity permutation in degree 5 
Lemma 3.2. Every multilinear polynomial identity of degree $n \leq 5$ satisfied by the symmetrization of the Jordan diproduct is a consequence of commutativity.

Proof. Similar to Lemma 3.1, but the matrix is much larger. In degree 5, the three association types $(((--)-)-)-,((--)(--))-,((--)-)(--)$ for a commutative nonassociative operation have respectively $60,15,30$ multilinear monomials. We expand the monomials with the identity permutation into the diassociative operad (Figure 2); there are $5 \cdot 5 !=600$ multilinear diassociative monomials. We construct the $600 \times 105$ matrix $E$ whose $(i, j)$ entry is the coefficient of the $i$-th diassociative monomial in the expansion of the $j$-th commutative nonassociative monomial. We extract the submatrix consisting of these 105 rows: $1-69,71,73-83,85,86,91,97-$ $101,103,121-125,127-129,133,145,147,149,151,169,241$. These rows are the lexicographically first subset which forms a basis of the row space. This submatrix has full rank and hence so does $E$, which completes the proof.

\section{Degree 6}

Theorem 4.1. In degree 6, there is an 8-dimensional space of multilinear polynomial identities satisfied by the symmetrization of the Jordan diproduct which do not follow from commutativity. This $S_{6}$-module has the structure $3[6] \oplus[51]$, where $[\lambda]$ denotes the simple module corresponding to partition $\lambda$, and so we may restrict our attention to nonlinear identities in the variables $x^{6}$ and $x^{5} y$. Every identity in the variables $x^{6}$ is a linear combination of these three identities:

$$
\begin{aligned}
& \left(\left(x^{2} x^{2}\right) x\right) x-2\left(\left(x^{2} x\right) x^{2}\right) x+4\left(\left(x^{2} x\right) x\right) x^{2}-3\left(x^{2} x^{2}\right) x^{2} \equiv 0, \\
& 2\left(\left(x^{2} x^{2}\right) x\right) x-2\left(\left(x^{2} x\right) x^{2}\right) x-2\left(\left(x^{2} x\right) x\right) x^{2}+\left(x^{2} x^{2}\right) x^{2}+\left(x^{2} x\right)\left(x^{2} x\right) \equiv 0, \\
& 8\left(\left(\left(x^{2} x\right) x\right) x\right) x-4\left(\left(x^{2} x^{2}\right) x\right) x-5\left(\left(x^{2} x\right) x^{2}\right) x-\left(\left(x^{2} x\right) x\right) x^{2}-\left(x^{2} x^{2}\right) x^{2} \\
& \quad+3\left(x^{2} x\right)\left(x^{2} x\right) \equiv 0 .
\end{aligned}
$$

Every identity in $x^{5} y$ is a linear combination of the four identities in Figure 3 .

Proof. At this point, the matrices become so large that efficient computation requires modular arithmetic, and we use the large prime $p=1000003$.

There are six association types for a commutative nonassociative operation, with respectively $360,90,180,180,45,90$ multilinear monomials, for a total of 945 :

$$
\begin{array}{lll}
(((--)-)-)-)-, & (((--)(--))-)-, & (((--)-)(--))-, \\
(((--)-)-)(--), & ((--)(--))(--), & ((--)-)((--)-) .
\end{array}
$$

With arguments abcdef, the expansions of these association types into the diassociative operad (using the symmetrized Jordan diproduct) each have 192 terms (and are therefore omitted); the coefficients of the expansions belong respectively to the sets $\{1,2,4,8,16\},\{2,8,16\},\{2,4,16\},\{2,4,8\},\{4,8\},\{4,8\}$. There are $6 \cdot 6 !=4320$ multilinear diassociative monomials. We construct the $4320 \times 945$ matrix $E$ whose $(i, j)$ entry is the coefficient of the $i$-th diassociative monomial in the expansion of the $j$-th commutative nonassociative monomial. Using linear algebra over $\mathbb{F}_{p}$, we find that $\operatorname{rank}(E)=937$ and hence the nullspace has dimension 8 .

Let $N$ denote the unique $8 \times 945$ matrix in row canonical form $(\mathrm{RCF})$ over $\mathbb{F}_{p}$ whose row space is the nullspace of $E$. The entries of $N$ belong to the following (extremely short) list of 33 congruence classes: 0-3, 8, 13, 14, 250000-250002, 499996-500004, 500012, 749997-750004, 999998-1000002. This strongly suggests that if we had been able to do this calculation using rational arithmetic then the 


$$
\begin{aligned}
& \left(\left(x^{2} x^{2}\right) x\right) y+\left(\left(x^{2} x^{2}\right) y\right) x+4\left(\left(x^{2}(x y)\right) x\right) x-\left(\left(x^{2} x\right) x^{2}\right) y \\
& \quad-2\left(\left(x^{2} x\right)(x y)\right) x-\left(\left(x^{2} y\right) x^{2}\right) x-2\left(((x y) x) x^{2}\right) x-2\left(\left(x^{2} x\right) x\right)(x y) \\
& \quad-\left(\left(x^{2} x\right) y\right) x^{2}-\left(\left(x^{2} y\right) x\right) x^{2}-2(((x y) x) x) x^{2}+\left(x^{2} x^{2}\right)(x y) \\
& \quad+2\left(x^{2}(x y)\right) x^{2}+\left(x^{2} x\right)\left(x^{2} y\right)+2\left(x^{2} x\right)((x y) x) \equiv 0, \\
& 8\left(\left(\left(x^{2} x\right) x\right) y\right) x+8\left(\left(\left(x^{2} y\right) x\right) x\right) x-5\left(\left(x^{2} x^{2}\right) y\right) x-4\left(\left(x^{2}(x y)\right) x\right) x \\
& \quad-\left(\left(x^{2} x\right) x^{2}\right) y-6\left(\left(x^{2} x\right)(x y)\right) x-3\left(\left(x^{2} y\right) x^{2}\right) x+2\left(\left(x^{2} x\right) x\right)(x y) \\
& \quad+\left(\left(x^{2} x\right) y\right) x^{2}-3\left(\left(x^{2} y\right) x\right) x^{2}+4(((x y) x) x) x^{2}-2\left(x^{2} x^{2}\right)(x y) \\
& \quad-4\left(x^{2}(x y)\right) x^{2}+3\left(x^{2} x\right)\left(x^{2} y\right)+2\left(x^{2} x\right)((x y) x) \equiv 0, \\
& 2\left(\left(x^{2} x^{2}\right) x\right) y+2\left(\left(x^{2} x^{2}\right) y\right) x+8\left(\left(x^{2}(x y)\right) x\right) x-3\left(\left(x^{2} x\right) x^{2}\right) y \\
& \quad-6\left(\left(x^{2} x\right)(x y)\right) x-3\left(\left(x^{2} y\right) x^{2}\right) x-6\left(((x y) x) x^{2}\right) x+6\left(\left(x^{2} x\right) x\right)(x y) \\
& \quad+3\left(\left(x^{2} x\right) y\right) x^{2}+3\left(\left(x^{2} y\right) x\right) x^{2}+6(((x y) x) x) x^{2}-5\left(x^{2} x^{2}\right)(x y) \\
& \quad-10\left(x^{2}(x y)\right) x^{2}+\left(x^{2} x\right)\left(x^{2} y\right)+2\left(x^{2} x\right)((x y) x) \equiv 0, \\
& 8\left(\left(\left(x^{2} x\right) x\right) x\right) y+8\left(\left(\left(x^{2} x\right) y\right) x\right) x+16((((x y) x) x) x) x-5\left(\left(x^{2} x^{2}\right) x\right) y \\
& \quad-16\left(\left(x^{2}(x y)\right) x\right) x-3\left(\left(x^{2} x\right) x^{2}\right) y-2\left(\left(x^{2} x\right)(x y)\right) x-\left(\left(x^{2} y\right) x^{2}\right) x \\
& \quad-8\left(((x y) x) x^{2}\right) x-2\left(\left(x^{2} x\right) x\right)(x y)-\left(\left(x^{2} x\right) y\right) x^{2}+3\left(\left(x^{2} y\right) x\right) x^{2} \\
& \quad-4(((x y) x) x) x^{2}-\left(x^{2} x^{2}\right)(x y)-2\left(x^{2}(x y)\right) x^{2}+2\left(x^{2} x\right)\left(x^{2} y\right) \\
& \quad+8\left(x^{2} x\right)((x y) x) \equiv 0 .
\end{aligned}
$$

Figure 3. Four identities in the variables $x^{5} y$

denominators would all be divisors of 4 . We multiply each row of $N$ by 4 , express the entries using symmetric representatives modulo $p$, interpret these symmetric representatives as integers, and divide each row by the GCD of its entries. The results appear in Figure 4, where column 2 gives the number of nonzero entries for each row; since these numbers are so large, it is not practical to display the corresponding multilinear polynomial identities.

\begin{tabular}{ccl} 
row & nonzero & reconstructed integer entries \\
\hline 1 & 693 & $-18,-17,-14,-13,-12,-4,0,1,2,3,4,5,6,8,10,42$ \\
2 & 660 & $-10,-8,-4,-3,-2,0,1,2,4,10$ \\
3 & 711 & $-8,-6,-4,-3,-2,-1,0,1,2,3,4$ \\
4 & 684 & $-22,-21,-20,-18,-17,-16,-4,-2,0,1,3,4,5,6,7,8,52$ \\
5 & 585 & $-9,-8,-5,-4,-3,-1,0,2,3,4,5,12$ \\
6 & 585 & $-9,-8,-5,-4,-3,-1,0,2,3,4,5,12$ \\
7 & 405 & $-3,0,1,8$ \\
8 & 495 & $-5,0,1,14$
\end{tabular}

FIGURE 4. Integer coefficients reconstructed from modular computations

The symmetric group $S_{6}$ acts on the row space of $N$ by permuting abcdef. For the partitions $1^{6}, 21^{4}, 2^{2} 1^{2}, 2^{3}, 31^{3}, 321,3^{2}, 41^{2}, 42,51,6$ we choose the following 


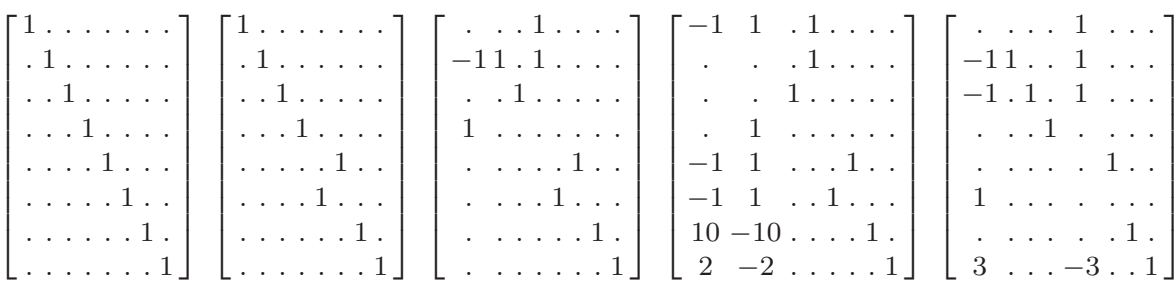

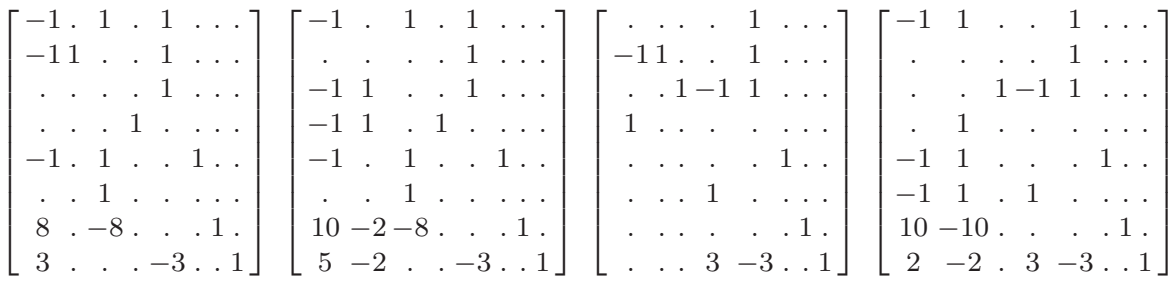

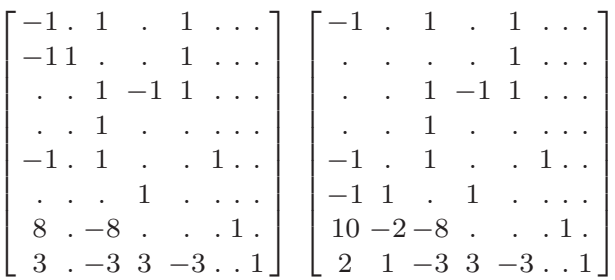

FiguRE 5. Representation matrices for conjugacy class representatives

conjugacy class representatives, expressed as products of disjoint cycles: e, (12), (12)(34), (12)(34)(56), (123), (123)(45), (123)(456), (1234), (1234)(56), (12345), (123456). For each of these 11 permutations $\sigma$, we compute the matrix representing $\sigma$ with respect to the reconstructed integer basis of the row space of $N$. These 11 matrices are displayed in Figure 5 . The traces of these matrices give the character of the row space of $N$ as an $S_{6}$-module: $[8,6,4,2,5,3,2,4,2,3,2]$. This character is a linear combination of the first two rows of the character table for $S_{6}$ :

$$
3[1,1,1,1,1,1,1,1,1,1,1]+[5,3,1,-1,2,0,-1,1,-1,0,-1] .
$$

Hence the row space of $N$ is isomorphic to $3[6] \oplus[51]$ as an $S_{6}$-module.

We now consider the problem of finding all nonlinear identities in the variables $x^{6}$ and $x^{5} y$. The structure of the row space of $N$ as an $S_{6}$-module implies that every multilinear identity in degree 6 can be expressed as a linear combination of linearizations of nonlinear identities corresponding to these two partitions. Since the matrices are small, we are able to use integer arithmetic.

$$
E=\left[\begin{array}{rrrrrr}
342 & 336 & 312 & 216 & 192 & 192 \\
118 & 112 & 104 & 168 & 192 & 128 \\
52 & 64 & 96 & 128 & 128 & 192 \\
52 & 64 & 96 & 128 & 128 & 192 \\
118 & 112 & 104 & 168 & 192 & 128 \\
342 & 336 & 312 & 216 & 192 & 192
\end{array}\right] \quad U=\left[\begin{array}{rrrrrr}
1 & -2 & 0 & -2 & -1 & 5 \\
-4 & 1 & 2 & 1 & -2 & 3 \\
0 & -1 & 0 & -2 & -1 & 5 \\
0 & 2 & -2 & -2 & 1 & 1 \\
0 & 1 & -2 & 4 & -3 & 0 \\
-8 & 4 & 5 & 1 & 1 & -3
\end{array}\right]
$$

FiguRE 6. Expansion matrix and transform matrix for variables $x^{6}$ 
With variables $x^{6}$, there is only one commutative nonassociative monomial for each of the six association types, and only one diassociative monomial for each possible center; we obtain the expansion matrix $E$ of Figure 6 Using elementary row operations defined over $\mathbb{Z}$, together with the LLL algorithm for lattice basis reduction [7, we compute the integer matrix $U$ of Figure 6 for which $\operatorname{det}(U)= \pm 1$ and $U E^{t}=H$ where $H$ (not displayed) is the Hermite normal form (HNF) of the transpose of $E$. From this we find that $\operatorname{rank}(E)=3$ and that the last three rows of $U$ form a reduced basis for the integer nullspace of $E$. The corresponding polynomial identities are displayed in equations (10)-(3).

With variables $x^{5} y$, there are respectively $5,3,4,4,2,2$ commutative nonassociative monomials for each of the six association types, which we order as follows:

$$
\begin{array}{lllll}
\left(\left(\left(x^{2} x\right) x\right) x\right) y, & \left(\left(\left(x^{2} x\right) x\right) y\right) x, & \left(\left(\left(x^{2} x\right) y\right) x\right) x, & \left(\left(\left(x^{2} y\right) x\right) x\right) x, & ((((x y) x) x) x) x, \\
\left(\left(x^{2} x^{2}\right) x\right) y, & \left(\left(x^{2} x^{2}\right) y\right) x, & \left(\left(x^{2}(x y)\right) x\right) x, & \left(\left(x^{2} x\right) x^{2}\right) y, & \left(\left(x^{2} x\right)(x y)\right) x \\
\left(\left(x^{2} y\right) x^{2}\right) x, & \left(((x y) x) x^{2}\right) x, & \left(\left(x^{2} x\right) x\right)(x y), & \left(\left(x^{2} x\right) y\right) x^{2}, & \left(\left(x^{2} y\right) x\right) x^{2} \\
(((x y) x) x) x^{2}, & \left(x^{2} x^{2}\right)(x y), & \left(x^{2}(x y)\right) x^{2}, & \left(x^{2} x\right)\left(x^{2} y\right), & \left(x^{2} x\right)((x y) x) .
\end{array}
$$

There are 36 diassociative monomials, ordered by position of the center and then by the position of $y$. We obtain the expansion matrix $E$ of Figure 7 Proceeding as before, we find that $\operatorname{rank}(E)=4$ and that the last three rows of the transform matrix $U$ form a reduced basis for the integer nullspace of $E$. The corresponding polynomial identities are displayed in equations (4)-(7).

\section{Degree 7}

Theorem 5.1. In degree 7, there is a 570-dimensional space of multilinear polynomial identities satisfied by the symmetrization of the Jordan diproduct which do not follow from commutativity or the linearizations of the identities in Theorem 4.1. This $S_{7}$-module has the following structure:

$$
7[61] \oplus 6[52] \oplus 4\left[51^{2}\right] \oplus 5[43] \oplus 5[421] \oplus\left[41^{3}\right] \oplus 3\left[3^{2} 1\right] \oplus\left[32^{2}\right] \oplus\left[321^{2}\right] .
$$

Proof. We obtain this result by extension of the methods already described for degree $n \leq 6$. Owing to the large size of the matrices in degree 7 , we decompose the computation into subproblems corresponding to the irreducible representations of the symmetric group $S_{7}$. The theory and algorithms required for this application of representation theory to polynomial identities are explained in detail in the survey paper [6]. We provide a brief summary of the computations.

Step 1. There are 11 association types for a commutative nonassociative operation in degree 7 , on which we impose the reverse degree-lexicographical order:

$$
\begin{array}{lll}
(((((--)-)-)-)-)-, & ((((--))(--))-)-)-, & ((((--)-)(--))-)-, \\
((((--)-)-)(--))-, & (((--))(--))(--))-, & (((--)-)((--)-))-, \\
((((--)-)-)-)(--), & (((--)(--))-)(--), & (((--)-)(--))(--), \\
(((--)-)-)((--)-), & ((--)(--))((--)-) . &
\end{array}
$$

For each type $t$, there is a nonempty set of permutations $\sigma \in S_{7}$ of order 2 for which $t(\iota)=t(\sigma)$ as a result of the commutativity of the symmetrized Jordan diproduct, where $\iota$ is the identity permutation and $t(\sigma)$ denotes type $t$ applied to permutation $\sigma$ of (the subscripts of) the arguments $x_{1}, \ldots, x_{7}$. For example, the last type produces $\sigma=(12),(34),(13)(24),(56)$. We obtain 30 polynomial identities $t(\iota)-t(\sigma) \equiv 0$ in degree 7 , called the symmetries of the association types. 


\begin{tabular}{|c|c|c|c|c|c|c|c|c|c|c|c|c|c|c|c|c|c|c|c|}
\hline 86 & 128 & 64 & 32 & 16 & 80 & 128 & 32 & 56 & 64 & 64 & 32 & 44 & 64 & 32 & 10 & 32 & 32 & 48 & \\
\hline 0 & 22 & 96 & 80 & 72 & 0 & 16 & 80 & 0 & 76 & 16 & 72 & 44 & 0 & 32 & 48 & 32 & 32 & 0 & 48 \\
\hline 0 & 0 & 6 & 88 & 124 & 0 & 0 & 84 & 0 & 12 & 128 & 80 & 0 & 24 & 64 & 64 & 0 & 48 & 48 & 24 \\
\hline 0 & 0 & 64 & 82 & 98 & 0 & 0 & 84 & 0 & 64 & 24 & 80 & 0 & 64 & 40 & 56 & 0 & 48 & 32 & 32 \\
\hline 0 & 128 & 96 & 56 & 31 & 0 & 128 & 52 & 0 & 80 & 64 & 44 & 64 & 0 & 32 & 28 & 64 & 16 & 0 & 48 \\
\hline 256 & 64 & 16 & 4 & 1 & 256 & 64 & 4 & 256 & 16 & 16 & 4 & 64 & 64 & 16 & 4 & 64 & 16 & 64 & 16 \\
\hline 32 & 22 & 32 & 16 & 8 & 32 & 16 & 16 & 48 & 12 & 16 & 8 & 20 & 64 & 32 & 16 & 32 & 32 & 32 & 16 \\
\hline 0 & 10 & 12 & 32 & 32 & 0 & 16 & 24 & 0 & 20 & 16 & 24 & 20 & 0 & 32 & 48 & 32 & 32 & 0 & 32 \\
\hline 0 & 0 & 4 & 22 & 46 & 0 & 0 & 28 & 0 & 8 & 24 & 32 & 0 & 16 & 40 & 56 & 0 & 48 & 32 & 16 \\
\hline 0 & 0 & 32 & 34 & 26 & 0 & 0 & 28 & 0 & 16 & 24 & 24 & 0 & 64 & 40 & 32 & 0 & 48 & 32 & 16 \\
\hline 0 & 64 & 32 & 12 & 5 & 0 & 64 & 12 & 0 & 32 & 16 & 12 & 64 & 0 & 16 & 12 & 64 & 16 & 0 & 32 \\
\hline 86 & 22 & 6 & 2 & 1 & 80 & 16 & 4 & 56 & 16 & 8 & 4 & 64 & 24 & 8 & 4 & 64 & 16 & 32 & 16 \\
\hline 20 & 10 & 6 & 8 & 4 & 32 & 16 & 4 & 48 & 8 & 16 & 8 & 20 & 24 & 32 & 16 & 32 & 16 & 48 & 24 \\
\hline 0 & 10 & 8 & 6 & 14 & 0 & 16 & 12 & 0 & 20 & 8 & 24 & 20 & 0 & 8 & 40 & 32 & 16 & 0 & 48 \\
\hline 0 & 0 & 6 & 14 & 16 & 0 & 0 & 16 & 0 & 12 & 24 & 24 & 0 & 24 & 40 & 32 & 0 & 32 & 48 & 24 \\
\hline 0 & 0 & 16 & 16 & 10 & 0 & 0 & 16 & 0 & 16 & 32 & 16 & 0 & 64 & 32 & 16 & 0 & 32 & 64 & 16 \\
\hline 0 & 22 & 12 & 6 & 6 & 0 & 16 & 12 & 0 & 28 & 8 & 16 & 44 & 0 & 8 & 16 & 32 & 16 & 0 & 48 \\
\hline 32 & 10 & 4 & 2 & 2 & 32 & 16 & 4 & 48 & 12 & 8 & 8 & 44 & 16 & 8 & 8 & 32 & 16 & 32 & 32 \\
\hline 32 & 10 & 4 & 2 & 2 & 32 & 16 & 4 & 48 & 12 & 8 & 8 & 44 & 16 & 8 & 8 & 32 & 16 & 32 & 32 \\
\hline 0 & 22 & 12 & 6 & 6 & 0 & 16 & 12 & 0 & 28 & 8 & 16 & 44 & 0 & 8 & 16 & 32 & 16 & 0 & 48 \\
\hline 0 & 0 & 16 & 16 & 10 & 0 & 0 & 16 & 0 & 16 & 32 & 16 & 0 & 64 & 32 & 16 & 0 & 32 & 64 & 16 \\
\hline 0 & 0 & 6 & 14 & 16 & 0 & 0 & 16 & 0 & 12 & 24 & 24 & 0 & 24 & 40 & 32 & 0 & 32 & 48 & 24 \\
\hline 0 & 10 & 8 & 6 & 14 & 0 & 16 & 12 & 0 & 20 & 8 & 24 & 20 & 0 & 8 & 40 & 32 & 16 & 0 & 48 \\
\hline 20 & 10 & 6 & 8 & 4 & 32 & 16 & 4 & 48 & 8 & 16 & 8 & 20 & 24 & 32 & 16 & 32 & 16 & 48 & 24 \\
\hline 86 & 22 & 6 & 2 & 1 & 80 & 16 & 4 & 56 & 16 & 8 & 4 & 64 & 24 & 8 & 4 & 64 & 16 & 32 & 16 \\
\hline 0 & 64 & 32 & 12 & 5 & 0 & 64 & 12 & 0 & 32 & 16 & 12 & 64 & 0 & 16 & 12 & 64 & 16 & 0 & 32 \\
\hline 0 & 0 & 32 & 34 & 26 & 0 & 0 & 28 & 0 & 16 & 24 & 24 & 0 & 64 & 40 & 32 & 0 & 48 & 32 & 16 \\
\hline 0 & 0 & 4 & 22 & 46 & 0 & 0 & 28 & 0 & 8 & 24 & 32 & 0 & 16 & 40 & 56 & 0 & 48 & 32 & 16 \\
\hline 0 & 10 & 12 & 32 & 32 & 0 & 16 & 24 & 0 & 20 & 16 & 24 & 20 & 0 & 32 & 48 & 32 & 32 & 0 & 32 \\
\hline 32 & 22 & 32 & 16 & 8 & 32 & 16 & 16 & 48 & 12 & 16 & 8 & 20 & 64 & 32 & 16 & 32 & 32 & 32 & 16 \\
\hline 256 & 64 & 16 & 4 & 1 & 256 & 64 & 4 & 256 & 16 & 16 & 4 & 64 & 64 & 16 & 4 & 64 & 16 & 64 & 16 \\
\hline 0 & 128 & 96 & 56 & 31 & 0 & 128 & 52 & 0 & 80 & 64 & 44 & 64 & 0 & 32 & 28 & 64 & 16 & 0 & 48 \\
\hline 0 & 0 & 64 & 82 & 98 & 0 & 0 & 84 & 0 & 64 & 24 & 80 & 0 & 64 & 40 & 56 & 0 & 48 & 32 & 32 \\
\hline 0 & 0 & 6 & 88 & 124 & 0 & 0 & 84 & 0 & 12 & 128 & 80 & 0 & 24 & 64 & 64 & 0 & 48 & 48 & 24 \\
\hline 0 & 22 & 96 & 80 & 72 & 0 & 16 & 80 & 0 & 76 & 16 & 72 & 44 & 0 & 32 & 48 & 32 & 32 & 0 & 48 \\
\hline 86 & 128 & 64 & 32 & 16 & 80 & 128 & 32 & 56 & 64 & 64 & 32 & 44 & 64 & 32 & 16 & 32 & 32 & 48 & 24 \\
\hline
\end{tabular}

Figure 7. Expansion matrix $E$ for variables $x^{5} y$

Step 2. We linearize the nonlinear identities of Theorem 4.1. For variables $x^{6}$, we replace the six occurrences of $x$ in each term by $x_{1} \cdots x_{6}$, and sum over all permutations of the subscripts $1, \ldots, 6$. For variables $x^{5} y$, we replace the five occurrences of $x$ in each term by $x_{1} \cdots x_{5}$, sum over all permutations of subscripts $1, \ldots, 5$, and replace $y$ by $x_{6}$. In both cases, we straighten the resulting monomials using commutativity of the symmetrized Jordan diproduct: we replace each monomial by the lexicographically first representative of its equivalence class.

Step 3. Let $f\left(x_{1}, \ldots, x_{6}\right)$ be one of the seven linearized identities produced by the previous step. Each such $f$ produces seven multilinear identities in degree 7:

$$
f\left(x_{1}, \ldots, x_{i} x_{7}, \ldots, x_{6}\right) \quad(1 \leq i \leq 6), \quad f\left(x_{1}, \ldots, x_{6}\right) x_{7} .
$$


We obtain 49 polynomial identities $g \equiv 0$ in degree 7 , called the consequences of the known identities in degree 6.

Step 4. For each of the 15 partitions $\lambda$ of 7 with corresponding irreducible representation of dimension $d_{\lambda}$, we construct the $30 d_{\lambda} \times 11 d_{\lambda}$ matrix $S_{\lambda}$ in which the $(i, j)$ block of size $d_{\lambda}$ is the matrix in representation $\lambda$ for the element of the group algebra given by the term (if any) of symmetry $i$ in association type $j$. We call $\operatorname{rank}\left(S_{\lambda}\right)$ the rank of the symmetries for partition $\lambda$.

Step 5. For each partition $\lambda$ of 7 , we construct the $49 d_{\lambda} \times 11 d_{\lambda}$ matrix $C_{\lambda}$ in which the $(i, j)$ block is the matrix in representation $\lambda$ for the element of the group algebra given by the terms (if any) of consequence $i$ in association type $j$. We call $\operatorname{rank}\left(C_{\lambda}\right)$ the rank of the consequences for partition $\lambda$.

Step 6. For each partition $\lambda$ of 7 , we stack (combine vertically) $S_{\lambda}$ and $C_{\lambda}$ to obtain the $79 d_{\lambda} \times 11 d_{\lambda}$ matrix $S C_{\lambda}$. We call $\operatorname{rank}\left(S C_{\lambda}\right)$ the rank of the old identities for partition $\lambda$, where old identities means identities in degree 7 which are consequences of known identities of lower degree (the symmetries of the association types are the consequences of commutativity).

Step 7. For each partition $\lambda$ of 7 , we construct the full rank matrix $N_{\lambda}$ in row canonical form whose row space is the nullspace of the restriction of the expansion map $X$ to the isotypic component of the $S_{7}$-module Free(7) for representation $\lambda$. We call $\operatorname{rank}\left(N_{\lambda}\right)$ the rank of all identities for partition $\lambda$. Hence $N_{\lambda}$ has size $\operatorname{rank}\left(N_{\lambda}\right) \times 11 d_{\lambda}$, and the row space of $S C_{\lambda}$ is a subspace of the row space of $N_{\lambda}$. The difference new $(\lambda)=\operatorname{rank}\left(N_{\lambda}\right)-\operatorname{rank}\left(S C_{\lambda}\right)$ is always nonnegative; we call it the rank of the new identities for partition $\lambda$.

We summarize these computations in Figure 8, which completes the proof.

\begin{tabular}{l|rrrrrrrrrrrrrrr}
$\lambda$ & 7 & 61 & 52 & $51^{2}$ & 43 & 421 & $41^{3}$ & $3^{2} 1$ & $32^{2}$ & $321^{1}$ & $31^{4}$ & $2^{2} 1$ & $2^{2} 1^{3}$ & $21^{5}$ & $1^{7}$ \\
\hline $\operatorname{rank}\left(S_{\lambda}\right)$ & 0 & 31 & 94 & 120 & 105 & 295 & 190 & 185 & 186 & 335 & 155 & 134 & 145 & 65 & 11 \\
$\operatorname{rank}\left(S C_{\lambda}\right)$ & 7 & 40 & 100 & 122 & 106 & 296 & 190 & 185 & 186 & 335 & 155 & 134 & 145 & 65 & 11 \\
$\operatorname{rank}\left(N_{\lambda}\right)$ & 7 & 47 & 106 & 126 & 111 & 301 & 191 & 188 & 187 & 336 & 155 & 134 & 145 & 65 & 11 \\
\hline $\operatorname{new}(\lambda)$ & 0 & 7 & 6 & 4 & 5 & 5 & 1 & 3 & 1 & 1 & 0 & 0 & 0 & 0 & 0
\end{tabular}

FIGURE 8. Multiplicities of irreducible representations in degree 7

\section{REFERENCES}

[1] M. Aymon, P.-P. Grivel: Un théorème de Poincaré-Birkhoff-Witt pour les algèbres de Leibniz. Comm. Algebra 31 (2003), no. 2, 527-544.

[2] L. A. Bokut, Y. Chen, C. Liv: Gröbner-Shirshov bases for dialgebras. Internat. J. Algebra Comput. 20 (2010), no. 3, 391-415.

[3] M. BónA, P. Flajolet: Isomorphism and symmetries in random phylogenetic trees. J. Appl. Probab. 46 (2009), no. 4, 1005-1019.

[4] M. R. Bremner: On the definition of quasi-Jordan algebra. Comm. Algebra 38 (2010), no. 12, 4695-4704.

[5] M. R. Bremner, V. Dotsenko: Algebraic Operads: An Algorithmic Companion. CRC Press, Boca Raton, 2016.

[6] M. R. Bremner, S. Madariaga, L. A. Peresi: Structure theory for the group algebra of the symmetric group, with applications to polynomial identities for the octonions. Comment. Math. Univ. Carolin. 57 (2016), no. 4, 413-452.

[7] M. R. Bremner, L. A. Peresi: An application of lattice basis reduction to polynomial identities for algebraic structures. Linear Algebra Appl. 430 (2009), no. 2-3, 642-659. 
[8] M. R. Bremner, L. A. Peresi: Special identities for quasi-Jordan algebras. Comm. Algebra 39 (2011), no. 7, 2313-2337.

[9] C. M. Glennie: Some identities valid in special Jordan algebras but not valid in all Jordan algebras. Pacific J. Math. 16 (1966), 47-59.

[10] N. Jacobson: Structure and Representations of Jordan Algebras. Colloquium Publications, Vol. XXXIX. American Mathematical Society, Providence, 1968.

[11] P. S. Kolesnikov: Varieties of dialgebras and conformal algebras. Sibirsk. Mat. Zh. 49 (2008), no. 2, 322-339.

[12] P. S. Kolesnikov, V. Yu. Voronin: On special identities for dialgebras. Linear Multilinear Algebra 61 (2013), no. 3, 377-391.

[13] J.-L. LOdAY: Une version non commutative des algèbres de Lie: les algèbres de Leibniz. Enseign. Math. (2) 39 (1993), no. 3-4, 269-293.

[14] J.-L. Loday: Algèbres ayant deux opérations associatives (digèbres). C. R. Acad. Sci. Paris Sér. I Math. 321 (1995), no. 2, 141-146.

[15] J.-L. Loday: Dialgebras. Dialgebras and Related Operads, pages 7-66. Lecture Notes in Mathematics, 1763. Springer, Berlin, 2001.

[16] J.-L. Loday, T. Pirashvili: Universal enveloping algebras of Leibniz algebras and (co)homology. Math. Ann. 296 (1993), no. 1, 139-158.

[17] J.-L. Loday, B. Vallette: Algebraic Operads. Grundlehren der Mathematischen Wissenschaften, 346. Springer, Heidelberg, 2012.

[18] M. Markl, E. Remm: Algebras with one operation including Poisson and other Lieadmissible algebras. J. Algebra 299 (2006), no. 1, 171-189.

[19] M. Markl, S. Shnider, J. Stasheff: Operads in Algebra, Topology and Physics. Mathematical Surveys and Monographs, 96. American Mathematical Society, Providence, 2002.

[20] K. McCrimmon: A Taste of Jordan Algebras. Springer-Verlag, New York, 2004.

[21] J. M. Osborn: Varieties of algebras. Advances in Math. 8 (1972) 163-369.

[22] R. Velásquez, R. Felipe: Quasi-Jordan algebras. Comm. Algebra 36 (2008), no. 4, 15801602.

[23] V. Voronin: Special and exceptional Jordan dialgebras. J. Algebra Appl. 11 (2012), no. 2, 1250029, 23 pages.

[24] K. A. Zhevlakov, A. M. Slinko, I. P. Shestakov, A. I. Shirshov: Rings That Are Nearly Associative. Translated from the Russian by Harry F. Smith. Pure and Applied Mathematics, 104. Academic Press, New York-London, 1982.

Department of Mathematics and Statistics, University of Saskatchewan, Canada

E-mail address: bremner@math.usask.ca 